\title{
Synthesis of 1, 4- Dihydropyrano [2, 3-c] pyrazole Derivatives with no Catalyst Under Ultrasound Irradiation
}

\author{
WANG SHU-XIANG ${ }^{*}$, and WANG WEI, LI JI-TAI \\ *College of Chemistry and Environmental Science, Hebei University, \\ Key Laboratory of Analytical Science and Technology, \\ Baoding 071002, Hebei Province, P. R. China.
}

Received 24 January 2005; Revised 12 March; Accepted 25 March 2005

\begin{abstract}
A series of 6-amino-4-aryl-5-cyano-3-methyl-1-phenyl-1, 4-dihydropyrano $[2,3-c]$ pyrazoles were synthesized in $84 \%-95 \%$ yields by the condensation of aromatic aldehydes, malononitrile with 3-methyl-1-phenyl-2-pyrazolin -5-one with no catalyst under ultrasound irradiation.
\end{abstract}

Key words: pyrano[2, 3-c] pyrazole, 3-methyl-1-phenyl-2-pyrazolin-5-one, aromatic aldehydes, synthesis

\section{Introduction}

Recently, 4H-benzo-pyran derivatives have attracted strong interest because of their useful biological and pharmacological activities, such as anticoagulant, spasmolytic, diuretic, anticancer and antianaphylactin. ${ }^{1}$ Some 2 -amino- $4 H$-pyrans can be employed as photoactive materials. ${ }^{2}$ Furthermore, multisubstitutional $4 H$-pyrans also constitute a structural unit of some natural products. $^{3}$ In addition, substituted pyrazole and derivatives can be used as important pharmaceuticals and agricultural chemicals. Usually these compounds were synthesized by classical 
methods. ${ }^{4-5}$ Microwave has been used to synthesize $4 H$-benzo-pyran ${ }^{6}$ in shorter reaction time, using piperidine as catalyst. But, these methods suffer from drawbacks such as longer reaction times, high temperatures, or necessary catalysts.

Ultrasound irradiation has increasingly been used in organic synthesis in recent years. Compared with traditional methods, this method is more convenient and easily controlled. A large number of organic reactions can be carried out in higher yield, shorter reaction time and milder conditions under ultrasound irradiation ${ }^{7}$. Herein, we would like to report the synthesis of 6-amino-4-aryl-5-cyano-3-methyl-1-phenyl-1, 4- dihydropyrano [2, 3-c] pyrazoles (4) through the condensation of aromatic aldehydes (1), malononitrile(2) with 3-methyl-1phenyl-2-pyrazolin-5-one (3) under ultrasound irradiation (scheme 1).

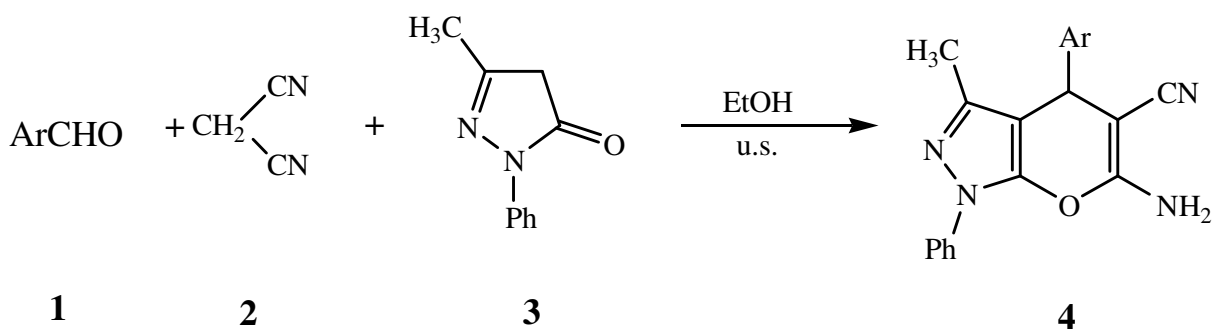

Scheme 1. Synthesis of 6-amino-4-aryl-5-cyano-3-methyl-1-phenyl-1, 4-dihydropyrano $[2,3-c]$ pyrazoles

\section{Experimental}

\section{Materials and Measurements}

Liquid aldehydes were purified by distillation prior to use. Melting points were uncorrected. IR spectra were recorded on a Bio-Rad FTS-40 spectrometer (KBr). ${ }^{1} \mathrm{H}$ NMR spectra were measured on a Bruker AVANCE $(400 \mathrm{MHz})$ spectrometer using TMS as an internal standard and $\mathrm{CDCl}_{3}$ as a solvent. Elemental analysis measured on a HERAEUS (CHN, Rapid) analyzer. Sonication was performed in Shanghai Branson-CQX ultrasonic cleaner with a frequency of $25 \mathrm{KHz}$ and a nominal power $250 \mathrm{~W}$. The reaction flasks were located in the maximum energy area in the cleaner, where the surface of reactants is slightly lower than the level of the water. The reaction temperature was controlled by addition or removal of water from ultrasonic bath.

\section{General Procedure}

Aromatic aldehyde 1(1 mmol), malononitrile $2(1 \mathrm{mmol})$, 3-methyl-1-phenyl-2-pyrazolin-5one $3(1 \mathrm{mmol})$ and EtOH $(5 \mathrm{~mL})$ were mixed in a $50 \mathrm{~mL}$ tapered Pyrex flask. The mixture was irradiated in the water bath of the ultrasonic cleaner at the room temperature. The reaction was monitored by TLC. After completion of the reaction, the reaction mixture was poured into ice water $(20 \mathrm{~mL})$ and the resulting solid was filtered. The collected solid was washed with water and then recrystallized from $\mathrm{EtOH} / \mathrm{CHCl}_{3}$ to afford pure products. All the products were confirmed by comparing their melting points, IR and ${ }^{1} \mathrm{H}$ NMR data with literature data. 
4c: IR (KBr) $v_{\max }: 3463,3319,2193,1655,1594,1391,1070,756 \mathrm{~cm}^{-1} ;{ }^{1} \mathrm{H}$ NMR $\delta: 1.93\left(\mathrm{~s}, 3 \mathrm{H}, \mathrm{CH}_{3}\right)$, 4.67(s, $1 \mathrm{H}, 4-\mathrm{H}), 4.74\left(\mathrm{~s}, 2 \mathrm{H}, \mathrm{NH}_{2}\right), 7.19-7.37(\mathrm{~m}, 5 \mathrm{H}, \operatorname{ArH}), 7.48(\mathrm{~d}, 2 \mathrm{H}, \mathrm{J}=8.0 \mathrm{~Hz}, \operatorname{ArH}), 7.67(\mathrm{~d}, 2 \mathrm{H}$, $\mathrm{J}=8.0 \mathrm{~Hz}, \mathrm{ArH})$. Found: $\mathrm{C}$ 66.01, H 4.05, N 15.41. Calc. for $\mathrm{C}_{20} \mathrm{H}_{15} \mathrm{ClN}_{4} \mathrm{O}: \mathrm{C} 66.21, \mathrm{H} 4.17, \mathrm{~N}$ 15.44.

4d: IR (KBr) $v_{\max }: 3472,3324,2194,1655,1591,1389,1264,1125,1029,752 \mathrm{~cm}^{-1}$; ${ }^{1} \mathrm{H}$ NMR $\delta: 1.91$ (s, $\left.3 \mathrm{H}, \mathrm{CH}_{3}\right), 4.73\left(\mathrm{~s}, 2 \mathrm{H}, \mathrm{NH}_{2}\right), 5.32(\mathrm{~s}, 1 \mathrm{H}, 4-\mathrm{H}), 7.21-7.43(\mathrm{~m}, 5 \mathrm{H}, \mathrm{ArH}), 7.48(\mathrm{~d}, 2 \mathrm{H}, \mathrm{J}=8.0 \mathrm{~Hz}, \mathrm{ArH})$, 7.67(d, 2H, J=8.0Hz, ArH). Found: C 66.11, H 4.08, N 15.61. Calc. for $\mathrm{C}_{20} \mathrm{H}_{15} \mathrm{ClN}_{4} \mathrm{O}: \mathrm{C} 66.21, \mathrm{H} 4.17$, N 15.44 .

4e: IR (KBr) $v_{\max }: 3456,3321,2198,1660,1591,1392,1269,1126,1049,758 \mathrm{~cm}^{-1} ;{ }^{1} \mathrm{H}$ NMR $\delta: 1.78$ (s, $\left.3 \mathrm{H}, \mathrm{CH}_{3}\right), 5.12(\mathrm{~s}, 1 \mathrm{H}, 4-\mathrm{H}), 6.73\left(\mathrm{~s}, 2 \mathrm{H}, \mathrm{NH}_{2}\right), 7.31-7.78(\mathrm{~m}, 8 \mathrm{H}, \mathrm{ArH})$. Found: $\mathrm{C} 66.35, \mathrm{H} 3.40, \mathrm{~N}$ 14.33. Calc. for $\mathrm{C}_{20} \mathrm{H}_{14} \mathrm{Cl}_{2} \mathrm{~N}_{4} \mathrm{O}: \mathrm{C} 60.47, \mathrm{H} 3.55, \mathrm{~N} 14.10$.

4f: IR (KBr) $v_{\text {max }}: 3072,2908,2258,1612,1528,1412,1194,1107,857,752 \mathrm{~cm}^{-1}$; ${ }^{1} \mathrm{H}$ NMR $\delta: 2.12$ (s, $\left.3 \mathrm{H}, \mathrm{CH}_{3}\right), 4.90\left(\mathrm{~s}, 2 \mathrm{H}, \mathrm{NH}_{2}\right), 5.22(\mathrm{~s}, 1 \mathrm{H}, 4-\mathrm{H}), 7.22-8.30(\mathrm{~m}, 9 \mathrm{H}, \mathrm{ArH})$. Found: C 64.40, H 3.12, N 18.80. Calc. for $\mathrm{C}_{20} \mathrm{H}_{15} \mathrm{~N}_{5} \mathrm{O}_{3}$ : C 64.34, H 3.05, N 18.76 .

4i: IR (KBr) $v_{\max }: 3414,3314,2179,1657,1593,1399,1257,1127,1026,753 \mathrm{~cm}^{-1} ;{ }^{1} \mathrm{H}$ NMR $\delta: 1.78$ (s, $\left.3 \mathrm{H}, \mathrm{CH}_{3}\right), 2.28\left(\mathrm{~s}, 3 \mathrm{H}, \mathrm{CH}_{3}\right), 4.62(\mathrm{~s}, 1 \mathrm{H}, 4-\mathrm{H}), 6.96\left(\mathrm{~s}, 2 \mathrm{H}, \mathrm{NH}_{2}\right), 7.02(\mathrm{~d}, 2 \mathrm{H}, \mathrm{J}=8.4 \mathrm{~Hz}, \mathrm{ArH}), 7.08$ $(\mathrm{d}, 2 \mathrm{H}, \mathrm{J}=8.4 \mathrm{~Hz}, \operatorname{ArH}), 7.29-7.33(\mathrm{~m}, 1 \mathrm{H}, \operatorname{ArH}), 7.46(\mathrm{~d}, 2 \mathrm{H}, \mathrm{J}=8.0 \mathrm{~Hz}, \operatorname{ArH}), 7.78(\mathrm{~d}, 2 \mathrm{H}, \mathrm{J}=8.0 \mathrm{~Hz}$, ArH). Found: C 73.52, H 5.19, N 16.25. Calc. for $\mathrm{C}_{21} \mathrm{H}_{18} \mathrm{~N}_{4} \mathrm{O}: \mathrm{C}$ 73.67, H 5.30, N 16.36.

4k: IR (KBr) $v_{\max }: 3414,3314,2179,1657,1593,1399,1257,1127,1026,753 \mathrm{~cm}^{-1} ;{ }^{1} \mathrm{H}$ NMR $\delta: 1.79$ (s, $\left.3 \mathrm{H}, \mathrm{CH}_{3}\right), 4.56(\mathrm{~s}, 1 \mathrm{H}, 4-\mathrm{H}), 6.72(\mathrm{~d}, 2 \mathrm{H}, \mathrm{J}=8.4 \mathrm{~Hz}, \operatorname{ArH}), 7.04(\mathrm{~d}, 2 \mathrm{H}, \mathrm{J}=8.4 \mathrm{~Hz}, \operatorname{ArH}) 7.12(\mathrm{~s}, 2 \mathrm{H}$, $\left.\mathrm{NH}_{2}\right), 7.29-7.33(\mathrm{~m}, 1 \mathrm{H}, \mathrm{ArH}), 7.49(\mathrm{~d}, 2 \mathrm{H}, \mathrm{J}=8.0 \mathrm{~Hz}, \mathrm{ArH}), 7.78(\mathrm{~d}, 2 \mathrm{H}, \mathrm{J}=8.0 \mathrm{~Hz}, \mathrm{ArH})$. Found: $\mathrm{C}$ 69.54, H 4.35, N 15.93. Calc. for $\mathrm{C}_{20} \mathrm{H}_{16} \mathrm{~N}_{4} \mathrm{O}_{2}: \mathrm{C} 69.76, \mathrm{H} 4.68, \mathrm{~N} 16.27$.

41: IR (KBr): $v_{\max }: 3400,3320,2198,1661,1595,1393,1254,1129,1037,789 \mathrm{~cm}^{-1}$; ${ }^{1} \mathrm{H}$ NMR $\delta: 2.12$ $\left(\mathrm{s}, 3 \mathrm{H}, \mathrm{CH}_{3}\right), 5.09(\mathrm{~s}, 1 \mathrm{H}, 4-\mathrm{H}), 5.95\left(2 \mathrm{H}, \mathrm{s}, \mathrm{OCH}_{2} \mathrm{O}\right), 6.92\left(\mathrm{~s}, 2 \mathrm{H}, \mathrm{NH}_{2}\right), 7.02-7.12(\mathrm{~m}, 2 \mathrm{H}, \mathrm{ArH})$, 7.15(m, $1 \mathrm{H}, \mathrm{ArH}), 7.17(\mathrm{~d}, 2 \mathrm{H}, \mathrm{J}=8.0 \mathrm{~Hz}, \mathrm{ArH}), 7.21(\mathrm{~m}, 1 \mathrm{H}, \mathrm{ArH}) 7.26(\mathrm{~d}, 2 \mathrm{H}, \mathrm{J}=8.0 \mathrm{~Hz}, \operatorname{ArH})$. Found: C 67.51, H 4.28, N 15.26. Calc. for $\mathrm{C}_{21} \mathrm{H}_{16} \mathrm{~N}_{4} \mathrm{O}_{3}: \mathrm{C} 67.73, \mathrm{H} 4.33, \mathrm{~N} 15.05$.

\section{Results and Discussion}

The results are summarized in Table 1. It can easily be seen that the condensation of 1,2 with 3 in EtOH gives 4 in good yields under ultrasound irradiation with no catalyst.

As shown in Table 1, the reaction of 1, 2 with 3 were carried out in 3 30min and give 4 in $84 \%$ 95\% yields using ethanol as solvent under ultrasound irradiation with no catalyst. We found that all aldehydes carrying electron-withdrawing $(1 \mathrm{f} \sim 1 \mathrm{~h})$ or weak electrondonating substituents (1a 1e, 1i) reacted with 2 and 3 very well at room temperature under ultrasound irradiation, gave corresponding products in good yields. However, these aldehydes carrying strong electron-donating substituents $(1 \mathrm{j} \sim 11)$ need longer time to finish the reaction. In addition, this reaction was affected by steric effect. The reaction need more time when aldehydes are 2 -substitted (such as $1 \mathrm{~d} \sim 1 \mathrm{f}$ ).

We proposed the possible mechanism to account for the reaction (described as Scheme 2). One molecule of 1 was firstly condensed with 2 to afford $\alpha$-cyanocinnamonitrile derivative (5). The step $(1+2 \rightarrow 5)$ could be regarded as a fast Knoevenagel reaction. The active methylene of 3 reacted with the electrophilic $\mathrm{C}=\mathrm{C}$ double bond of 5 to give the intermediate (6). Then the intermediate 6 was cyclized by the nucleophilic attack of $\mathrm{OH}$ group on the cyano $(\mathrm{CN})$ and gave the intermediate 7 . Finally the expected products 4 were afforded $(7 \rightarrow 8 \rightarrow 4)$. 
Table 1. Condensation of aromatic aldehydes, malononitrile with 3-methyl-1-phenyl-2pyrazolin-5-one in EtOH with no catalyst under ultrasound irradiation

\begin{tabular}{ccccc}
\hline Entry & $\mathrm{Ar}$ & Time/min & Yield*/\% & M.p. $/{ }^{\circ} \mathrm{C}(\mathrm{Lit})$. \\
\hline $\mathrm{a}$ & $\mathrm{C}_{6} \mathrm{H}_{5}$ & 5 & 84 & $167-169(168-170)^{6}$ \\
$\mathrm{~b}$ & $4-\mathrm{ClC}_{6} \mathrm{H}_{4}$ & 3 & 95 & $172-174(174-175)^{6}$ \\
$\mathrm{c}$ & $3-\mathrm{ClC}_{6} \mathrm{H}_{4}$ & 8 & 91 & $157-158$ \\
$\mathrm{~d}$ & $2-\mathrm{ClC}_{6} \mathrm{H}_{4}$ & 15 & 94 & $145-146$ \\
$\mathrm{e}$ & $2,4-\mathrm{Cl}_{2} \mathrm{C}_{6} \mathrm{H}_{4}$ & 10 & 92 & $182-184$ \\
$\mathrm{f}$ & $2-\mathrm{NO}_{2} \mathrm{C}_{6} \mathrm{H}_{4}$ & 20 & 87 & $157-158$ \\
$\mathrm{~g}$ & $3-\mathrm{NO}_{2} \mathrm{C}_{6} \mathrm{H}_{4}$ & 5 & 90 & $187-189(188-190)^{6}$ \\
$\mathrm{~h}$ & $4-\mathrm{NO}_{2} \mathrm{C}_{6} \mathrm{H}_{4}$ & 3 & 87 & $193-195(194-196)^{6}$ \\
$\mathrm{i}$ & $4-\mathrm{CH}_{3} \mathrm{C}_{6} \mathrm{H}_{4}$ & 5 & 86 & $176-178$ \\
$\mathrm{j}$ & $4-\mathrm{OCH}_{3} \mathrm{C}_{6} \mathrm{H}_{4}$ & 20 & 89 & $169-170(170-172)^{6}$ \\
k & $4-\mathrm{OHC}_{6} \mathrm{H}_{4}$ & 30 & 93 & $214-215$ \\
1 & $3,4-\mathrm{OCH}_{2} \mathrm{OC}_{6} \mathrm{H}_{3}$ & 25 & 90 & $175-176$
\end{tabular}

*Isolated yield, based on aldehydes.

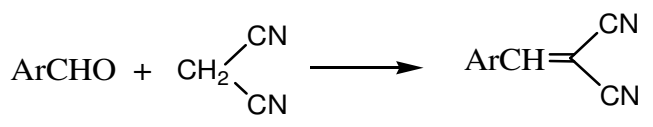
1
2
5

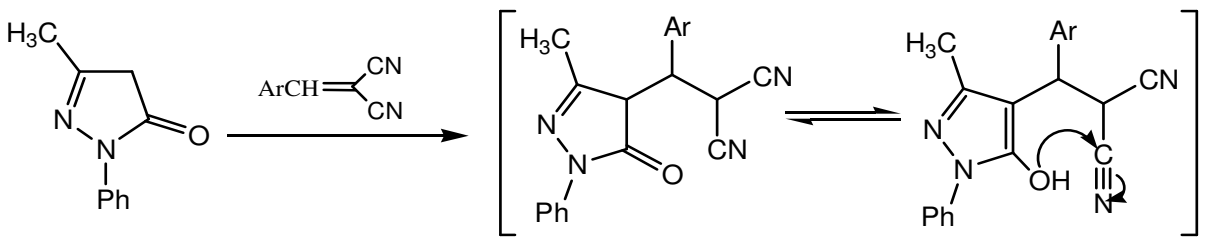

6

7<smiles>Cc1nn(-c2ccccc2)c2c1C([Al])C(C#N)C(=N)O2</smiles>

8<smiles>Cc1nn(-c2ccccc2)c2c1C(Br)C(C#N)=C(N)O2</smiles>

4

Scheme 2. The possible mechanism of the reaction 


\section{Conclusion}

In conclusion, we have described a simple and efficient procedure for the preparation of 6-amino-4aryl-5-cyano-3-methyl-1-phenyl-1,4-dihydropyrano [2, 3-c]pyrazoles in EtOH with no catalyst under ultrasound irradiation.

\section{Acknowlegment}

The Education commission of Hebei Povince (201104) and Natural Science Foundation of Hebei Province (203107), China, supported this project.

\section{References}

1 (a) Andreani L L and Lapi E, Boll. Chim. Farm. 1960, 99, 583; (b) Bonsignore L, Loy G, Secci D and Calignano A, Eur. J. Med.. Chem. 1993, 28, 517.

2 Armetso D, Horspool W M, Martin N, Ramos A and Seaone C, J. Org. Chem. 1989, 54, 3069.

3 Hatakeyama S, Ochi N, Numata H and Takano S J, Chem. Soc. Chem. Commun. 1988, 1202.

$4 \quad$ Kamaljit S, Jasbi S and Harjit S, Tetrahedron 1996, 52, 14273.

$5 \quad$ Abd Allah O A, Il Farmaco 2000, 55, 641.

6 Zhou J F, Tu S J, Gao Y and Qi M, Chin. J. Org. Chem. 2001, 21, 742. [Chem. Abstr., 2001, 136(8), 118416v].

7 (a) Mason T J, Practical Sonochemistry, Ellis Horwood, 1991, 18; (b) Luche J L, Synthetic Organic Sonochemistry, Plenum Press, New York, 1998, 3; (c) Li J T, Bian Y J, Zang H J and Li T S, Synth. Commun. 2002, 32, 547; (d) Li J T, Han J H, Yang J H and Li T S, Ultrason. Sonochem. 2002, 9, 237. 


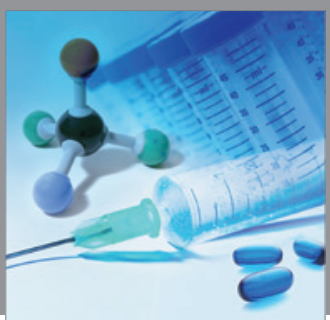

International Journal of

Medicinal Chemistry

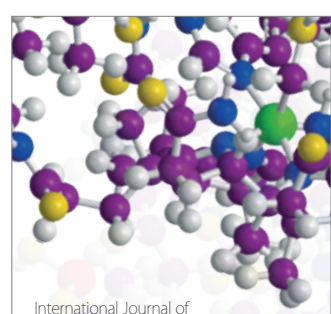

Carbohydrate Chemistry

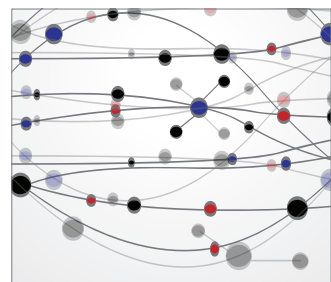

The Scientific World Journal
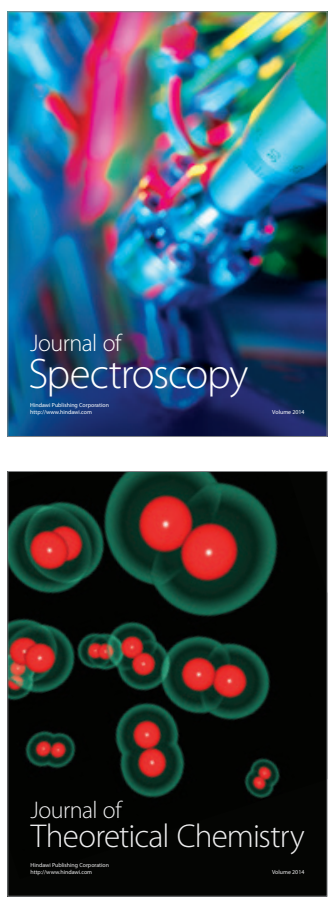
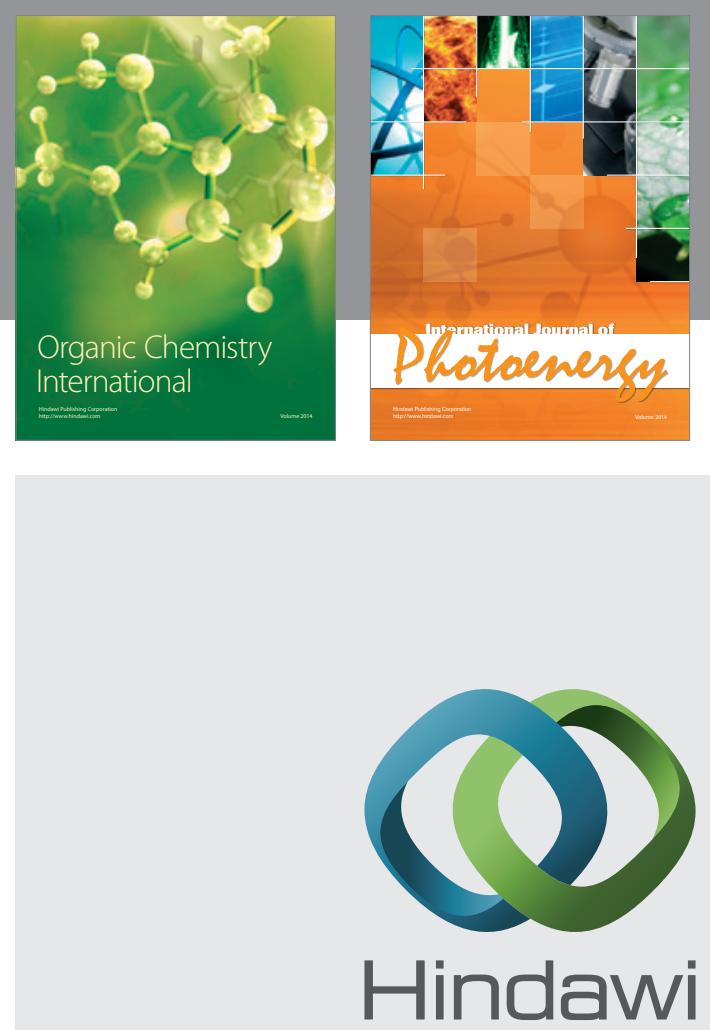

Submit your manuscripts at

http://www.hindawi.com
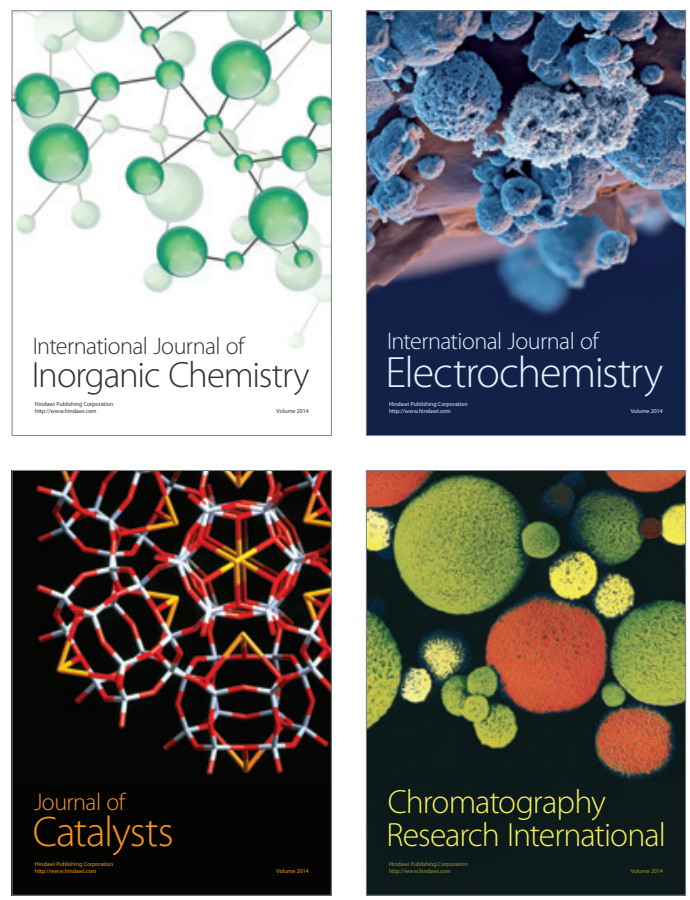
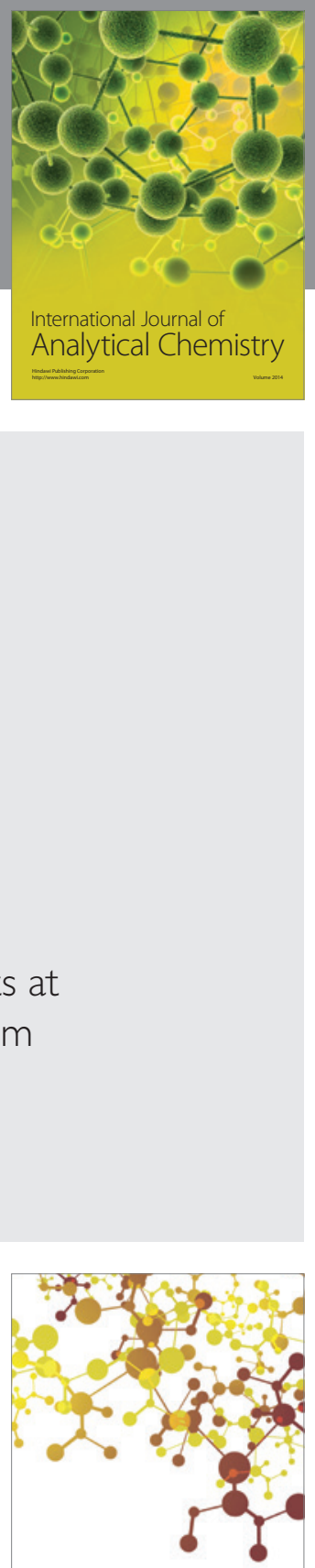

Journal of

Applied Chemistry
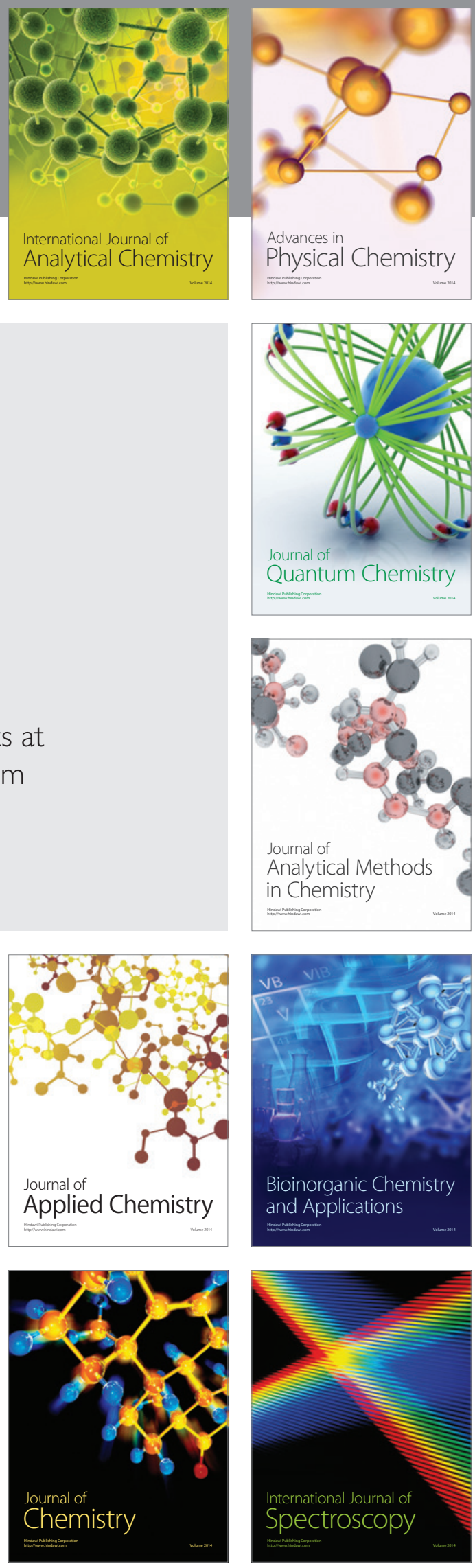